\title{
Energy Partitioning in Molecular Photodissociation: Methyl Nitrite Photolysis
}

\author{
F. LAHMANI, C. LARDEUX and D. SOLGADI
}

Laboratoire de Photophysique Moléculaire, Bât. 213, et Laboratoire d'Utilisation du Rayonnement Electromagnérique, Bât. 200, Université Paris-Sud, 91405Orsay

The photodissociation of methyl nitrite $\mathrm{CH}_{3} \mathrm{ONO}$ in three different electronically excited states has been studied by determining the internal state distribution of one of the photofragments. The fluorescence of NO $A^{2} \Sigma^{+}$produced for excitation of $\mathrm{CH}_{3} \mathrm{ONO}$ between 1200 and $1650 \AA$ and of $\mathrm{CH}_{3} \mathrm{O}^{2} A_{1}$ produced at $1930 \AA$ has been investigated. The vibrational distribution in both species can be explained by statistical considerations. The rotational excitation of $\mathrm{NO} A^{2} \Sigma^{+}$is of Boltzmann type. For $\mathrm{CH}_{3} \mathrm{ONO}$ excited in the first $n \pi^{*}$ excited state at $3550 \AA$ the NO $X$ fragment has been probed by a two photon laser excited fluorescence technique. The nascent NO $X v^{\prime \prime}=0,1,2,3$ exhibit a population inversion and a high degree of rotational excitation.

The study of the dissociative relaxation channels from electronic excited states of polyatomic molecules is of special interest in the development and understanding of molecular dynamics, secondary photochemical reactions, and new laser obtention. The most important question about these processes is related to the manner in which the excess energy is partitioned among the degrees of freedom of the primary products (relative motion and internal energy content).

While triatomic molecules provide the favored systems to test the current theories of photodissociation based on atom + diatom collision dynamics description, there is a need to investigate the behavior of larger polyatomic systems. For large molecules with numerous degrees of freedom a competition can be expected between fragmentation 
and vibrational redistribution and this raises the question of the extent of energy randomization within the initial excited molecule and how it affects the energy disposal in the products.

We present here some of the informations we have collected on the photodissociation of $\mathrm{CH}_{3} \mathrm{ONO}$ in different spectral regions. This molecule possesses a weak $\mathrm{O}-\mathrm{N}$ simple bond $\left(D_{0}=1.81 \mathrm{eV}\right)^{1}$ and traditional photochemical studies have established that the major primary process is the fission of the $\mathrm{O}-\mathrm{N}$ central bond into methoxy and NO fragments. Most of the photolysis work on organic nitrites have been concerned with the analysis of the final products in the near UV region and it has been found that, depending on the excitation wavelength, a substantial fraction of the alkoxy radical is sufficiently vibrationally hot to undergo a secondary spontaneous unimolecular decomposition. ${ }^{2}$ At higher excitation energy $\left(\lambda_{\text {exc }}<\right.$ $2000 \AA$ ) production of electronically excited $\mathrm{CH}_{3} \mathrm{O}^{3}$ and $\mathrm{NO}^{4}$ is energetically possible and has been observed. In this work we report results on the vibrational and rotational distribution of $\mathrm{NO} \mathrm{A}^{2} \Sigma^{+}$ produced in the VUV region compared with that of NO $X$ produced in the near UV region. In the first case direct detection and analysis of the fluorescence of $\mathrm{NO}$ allows to obtain internal state distribution information while in the second case the two photon laser fluorescence excitation spectra has been used to monitor the nascent NO $X$ product. On the other hand, the emission spectra of $\mathrm{CH}_{3} \mathrm{O}$ has been obtained in the laser photolysis of $\mathrm{CH}_{3} \mathrm{ONO}$ at $1930 \AA$ and the analysis of fluorescence gives insight into the vibrational energy disposal in the polyatomic fragment.

\section{EXPERIMENTAL}

$\mathrm{CH}_{3} \mathrm{ONO}$ was prepared from the reaction of sodium nitrite on methanol in a sulfuric acid medium. Its absorption spectrum (Figure 1) presents from 4000 to $1200 \AA$ three well separated regions. Between 3000 and $4000 \AA$ the absorption arises from a $n \rightarrow \pi^{*}$ transition and shows a vibrational structure broadened by predissociation and due to a progression in the $\mathrm{N}=\mathrm{O}$ stretching mode. At shorter wavelength a more intense wide absorption band with a maximum at $2150 \AA$ is assigned to an intramolecular charge transfer from the lone pair $\pi$ orbital of the $\mathrm{OCH}_{3}$ group to the $\pi^{*}$ orbital of 


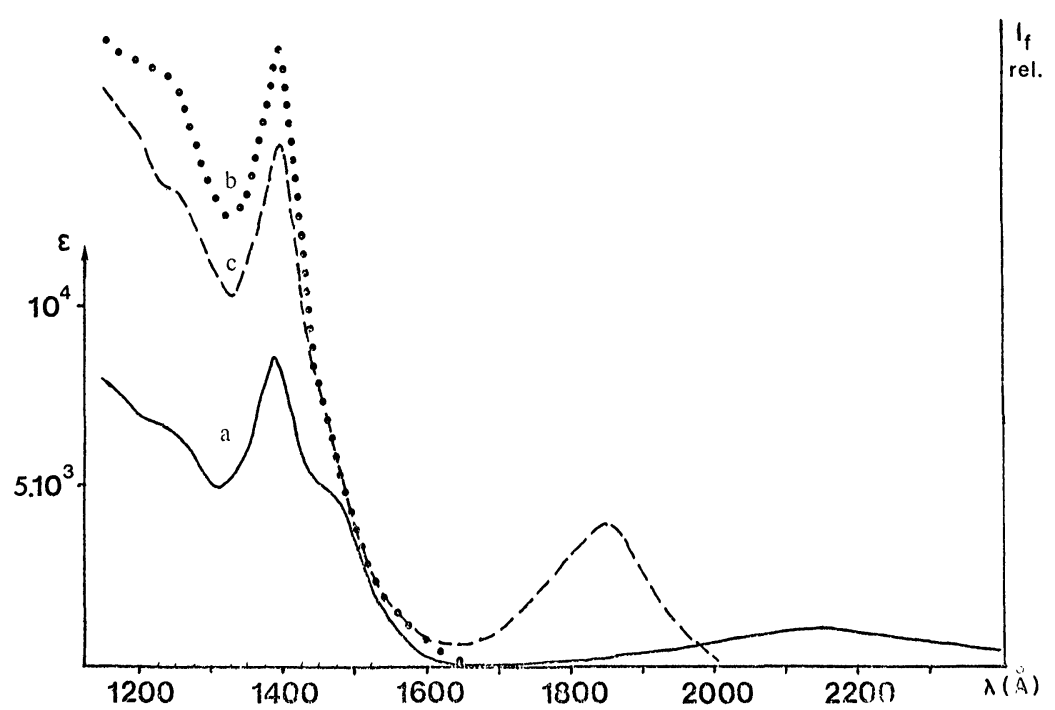

FIGURE 1 Absorption spectra (a) and global photofragment fluorescence excitation spectrum (b)(c) of methylnitrite between 1200 and $2400 \AA$. The curve (b) corresponds to the fluorescence emitted between 2000 and $3000 \AA$ (mainly NO $A^{2} \Sigma^{+}$) and the curve (c) to the fluorescence emitted between $3000 \AA$ and $6000 \AA$.

the $\mathrm{N}=\mathrm{O}$ group. In the vacuum ultraviolet the spectrum is composed of diffuse bands with a prominent peak at $1390 \AA .^{5}$ In this region Rydberg transitions $n \rightarrow 3 s$ and $n \rightarrow 3 p$ calculated at 1650,1480 and $1460 \AA$ can be overlapped by the $\pi \pi^{*}$ transition localized on the NO group.

Different excitation sources and experimental detection have been used to photodissociate $\mathrm{CH}_{3} \mathrm{ONO}$ in different spectral regions.

1) In the $1200-1650 \AA$ region the light from the synchrotron of the Orsay collision ring (ACO $\tau=1 \mathrm{~ns}, 13 \mathrm{MHz}$ repetition rate) was dispersed through a home made monochromator. The fluorescence was spectrally analyzed using a second low resolution monochromator. Photon counting techniques were used with an RTC 56 SB UVP photomultiplier for the detection. Some experiments have been performed with higher incident flux using a xenon resonance lamp to photolyse $\mathrm{CH}_{3} \mathrm{ONO}$ at $1470 \AA$ 
2) An Ar F excimer laser (Lambda Physik EMG 101) was used to photodissociate $\mathrm{CH}_{3} \mathrm{ONO}$ in the second absorption continuum at $1932 \AA$ and study directly the emission of $\mathrm{CH}_{3} \mathrm{O}$ which was dispersed through a $60 \mathrm{~cm}$ Jobin and Yvon monochromator and detected with an EMI 9635 photomultiplier and associated electronics

3) In the near UV region a small part of the third harmonic output of a Nd:YAG Laser (Quantel) operating at a repetition rate of $10 \mathrm{~Hz}$ provided $10 \mathrm{~ns}$ photolysis pulses at $3550 \AA$. The other part of the $3550 \AA$ laser beam pumped a dye laser which was tuned between $4500 \AA$ and $5000 \AA$ to excite the NO $X$ fragment to its $A^{2} \Sigma^{+}$state via a two photon transition. Its width was $0.1 \mathrm{~cm}^{-1}$. The two laser beams propagated collinearly in the fluorescence cell, the probe laser being delayed of about $20 \mathrm{~ns}$ after the UV beam. The fluorescence from NO $A$ was focused through Schott UG 5 and chlorine gas filters onto a solar blind photomultiplier (RTC 56 SB UV). The fluorescence signal was then amplified, integrated over $2 \mu \mathrm{s}$ after the pulse and recorded on a SEIN multichannel analyzer operating on the multiscale mode. The intensity of the dye laser was monitored by a photodiode placed after the photolysis cell and recorded simultaneously with the fluorescence.

\section{RESULTS AND DISCUSSION}

\section{VUV photolysis}

The fragmentation channel followed in the vacuum ultraviolet region is the production of NO in its first Rydberg state $A^{2} \Sigma^{+}$. From the fluorescence spectra it can be seen that NO $A^{2} \Sigma^{+}$is populated in different vibrational levels $v^{\prime}=0,1,2,3$. The energy dependence of the relative vibrational population has been determined by scanning the excitation wavelength and recording the gated fluorescence at different observation wavelength corresponding to the $(0-1),(1-0)$, $(2-0),(3-0)$ emission bands. The fluorescence intensity divided by the relative emission probability of the observed band and corrected for $\mathrm{CH}_{3} \mathrm{ONO}$ pressure quenching gives the vibrational distribution as a function of the excess energy available in the process. It can be deduced from this study that the $v^{\prime}=0$ is the most populated level, higher vibrational level population increasing smoothly with increas- 
ing excess energy. In order to analyse the vibrational energy disposal, experimental results have been compared to a priori distribution by assuming equal probabilities for all product quantum states allowed by the conservation of energy. The $N v^{\prime}=n / N v^{\prime}=0$ ratio is then related to the ratio $\rho(E, n) / \rho(E, 0)$ where $\rho(E, n)$ is the density of states which have a total energy $E$ with $n$ quanta in the NO vibration. The results plotted on Figure 2 as dashed lines show that NO $v^{\prime}=1$ and $v^{\prime}=2$ relative population although showing a comparative energy dependence are more populated than predicted on statistical grounds. This can be due to a non total energy randomization which would lead to the excitation of preferential modes in the $\mathrm{CH}_{3} \mathrm{O}$ fragment. On the other hand a rotational analysis has been made for NO $A$ $\left(v^{\prime}=0\right)$ produced by the $1470 \AA$ photolysis of $\mathrm{CH}_{3} \mathrm{ONO}$. The experimental spectrum can be reproduced by a simulated spectrum calculated with a Boltzmann distribution of $J$ levels in NO $A\left(v^{\prime}=0\right)$ peaking at $J_{\max }=9.5$ corresponding to a rotational temperature of $600 \mathrm{~K}$. A Boltzmann distribution is expected if the conservation of angular momentum is the critical constraint and if the orbital angular momentum of the separating fragments is small (i.e., for a dissociation from a near linear configuration). In that case the angular moment of the $\mathrm{CH}_{3} \mathrm{ONO}$ molecule at room temperature $\left(J_{\max }=26\right)$ should

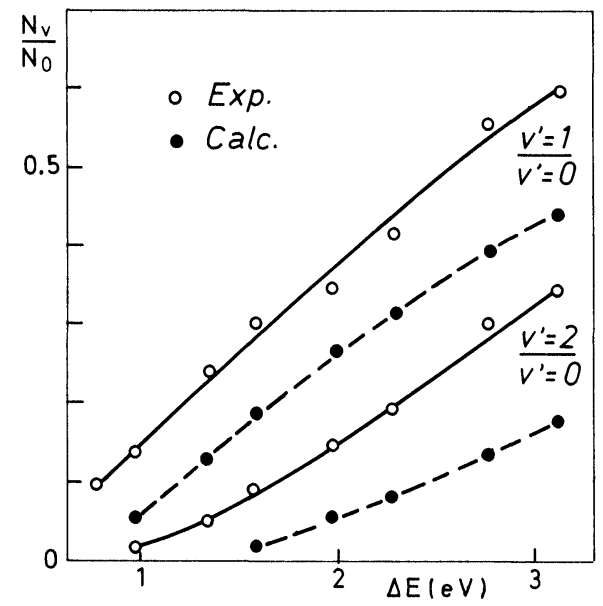

FIGURE 2 Relative population of NO $A v^{\prime}=1 / v^{\prime}=0$ and $v^{\prime}=2 / v^{\prime}=0$ as a function of excess energy. The dashed lines give the ratios expected on statistical grounds. 
be partitioned between those of the fragments. As $J_{\max \text { NO }}=9.5$ this would lead to $J_{\max \mathrm{CH}_{3} \mathrm{O}}=16.5$ which corresponds to the same rotational energy as in NO $\left(T_{\text {rot }}=600 \mathrm{~K}\right)$.

\section{$1930 \AA$ photolysis}

The excitation spectrum of the global photofragment fluorescence from $\mathrm{CH}_{3} \mathrm{ONO}$ dissociation shows a band starting at $2100 \AA$ with a maximum around $1850 \AA$ (see Figure 1 ). The corresponding emitting species has been previously identified as the $\mathrm{CH}_{3} \mathrm{O} A^{2} A_{1}$ state. ${ }^{3}$ The dispersed fluorescence of excited $\mathrm{CH}_{3} \mathrm{O}$ produced by photolysis of $\mathrm{CH}_{3} \mathrm{ONO}$ at $1930 \AA$ shows a very diffuse and overlapped structure which prevents any clear assignments of the vibrational states populated in $\mathrm{CH}_{3} \mathrm{O}$ (Figure 3a). However addition of helium (0.1-10 Torr) to the nitrite makes the vibrational structure sharper and simpler to assign. From the helium pressure effect it can be deduced that collisions relax predominantly the rotational excitation without affecting the relative vibrational population of $\mathrm{CH}_{3} \mathrm{O} A^{2} A_{1}$. The high energy part of the fluorescence spectrum exhibits bands spaced by $650 \mathrm{~cm}^{-1}$ originating from $v^{\prime}=4,3,2,1,0$ of the $\mathrm{C}-\mathrm{O}$ stretching mode of $\mathrm{CH}_{3} \mathrm{O}$. The vibrational distribution for this active mode can thus be estimated using the known Franck-Condon factors. ${ }^{6}$ The results show that the vibrational population decreases exponentially with increasing vibrational energy (Figure 4). Such a behaviour is expected under the assumption of statistical redistribution of the excess energy.

\section{$3550 \AA$ Å photolysis}

The study of energy disposal in NO $X$ resulting from photodissociation at $3550 \AA$ using a two photon laser excitation technique is under development. The NO $X v^{\prime}=0,1,2,3$ have been detected by probing the $X, v^{\prime \prime} \rightarrow A v^{\prime}=0 \rightarrow 0,1 \rightarrow 0,2 \rightarrow 0,3 \rightarrow 0$ transitions. A part of typical spectra are shown in Figure 5 for $v^{\prime \prime}=1 \rightarrow v^{\prime}=0$. The most striking observation is that NO $X$ exhibits extensive rotational excitation that do not correspond to a Boltzmann distribution of $J$ levels. In fact one can note the absence of low $J$. level population and of prominent band heads while $J$ levels between 20.5 and 40.5 can be easily identified in the $\mathrm{O}_{12}, \mathrm{O}_{22}+\mathrm{P}_{12}$ and $\mathrm{O}_{11}$ branches. Some simulations of the spectra have been attempted using the Hönl-London 

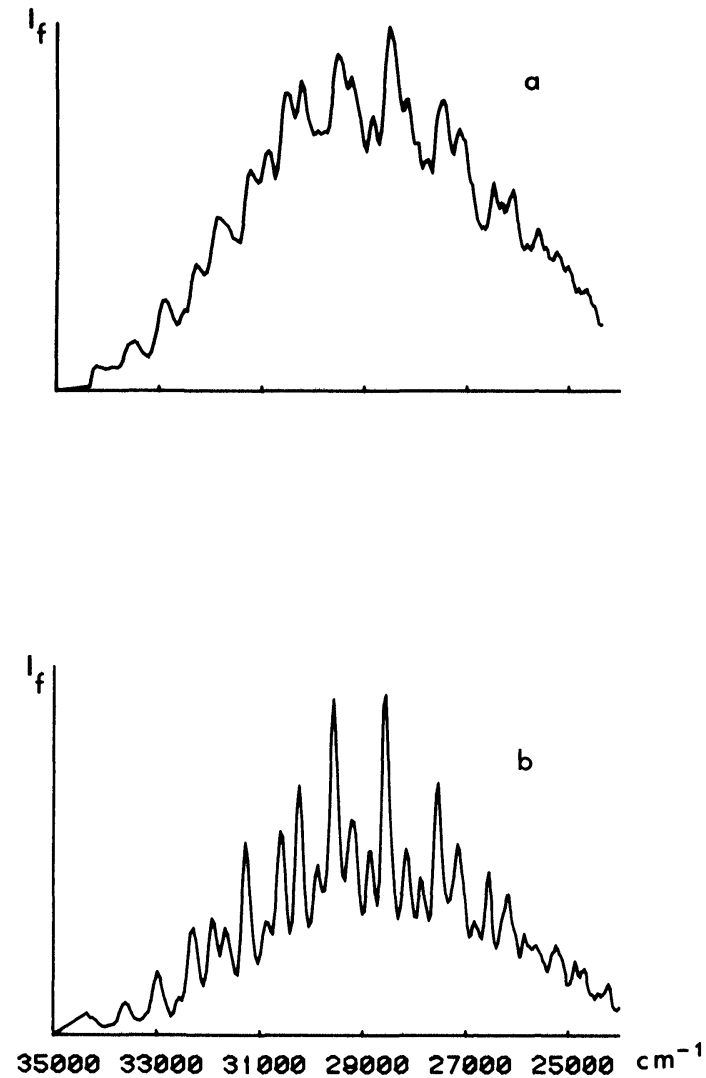

FIGURE 3 Fluorescence of $\mathrm{CH}_{3} \mathrm{OA} \mathrm{A}^{2} \mathrm{~A}_{1}$ obtained in the photolysis of $\mathrm{CH}_{3} \mathrm{ONO}$ at $1930 \AA$ : (a) $3 \mathrm{mT}$ Torr of $\mathrm{CH}_{3} \mathrm{ONO}$; (b) 3 mTorr of $\mathrm{CH}_{3} \mathrm{ONO}+3$ Torr of $\mathrm{He}$.

factors for the NO two photon absorption given by Halpern et al. ${ }^{7}$ This calculation corroborates the observation that the rotational distributions are definitely not of Boltzmann type. On the other hand, in order to estimate the relative vibrational population the excitation spectra of the sequence $v^{\prime \prime} \rightarrow v^{\prime}=0 \rightarrow 0,1 \rightarrow 1,2 \rightarrow 2$ have been recorded for $\mathrm{CH}_{3} \mathrm{ONO}$ in the presence of argon in order to thermalize the rotational populations. The preliminary estimation indicates that NO $X v^{\prime \prime}=1$ is more populated than $v^{\prime \prime}=0$ by a factor of $\sim 2$. 


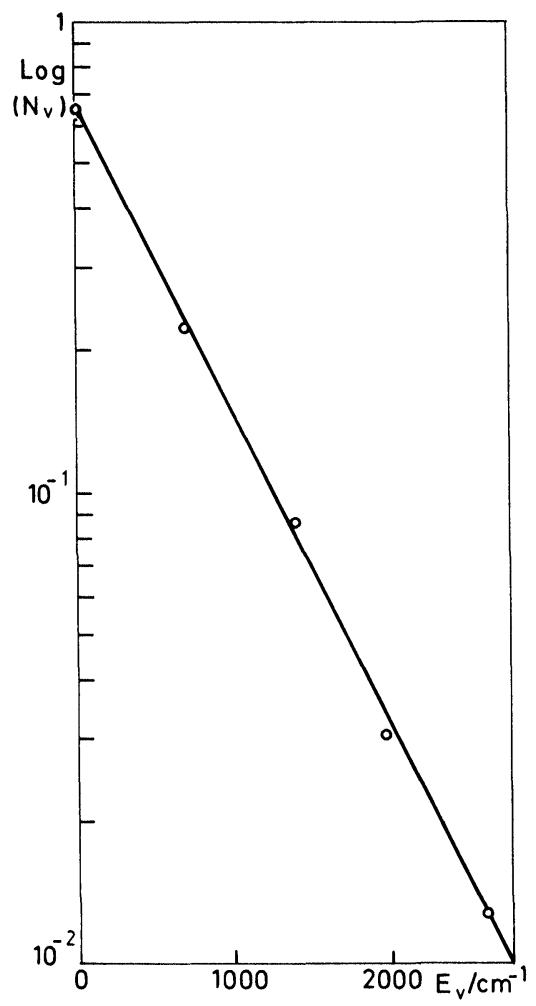

FIGURE 4 Semi logarithmic plot of the vibrational population in the $\mathrm{C}-\mathrm{O}$ stretching mode of $\mathrm{CH}_{3} \mathrm{O}^{2} A_{1}$ from photodissociation of $\mathrm{CH}_{3} \mathrm{ONO}$ at $1930 \AA$.

\section{CONCLUSION}

The photodissociation of $\mathrm{CH}_{3} \mathrm{ONO}$ has been studied in different electronic states by probing the internal energy of one of the two fragments. While the vibrational distribution in NO $A^{2} \Sigma^{+}$in the vacuum $U V$ region and $\mathrm{CH}_{3} \mathrm{O} A^{1} A_{1}$ can be interpreted the basis of statistical considerations (with possible deviations of the total randomization assumption) the results on $\mathrm{CH}_{3} \mathrm{ONO}$ excited in the first excited state indicate a completely different behavior. The same contrast is observed for the rotational distribution in NO $A$ at $1470 \AA$ 


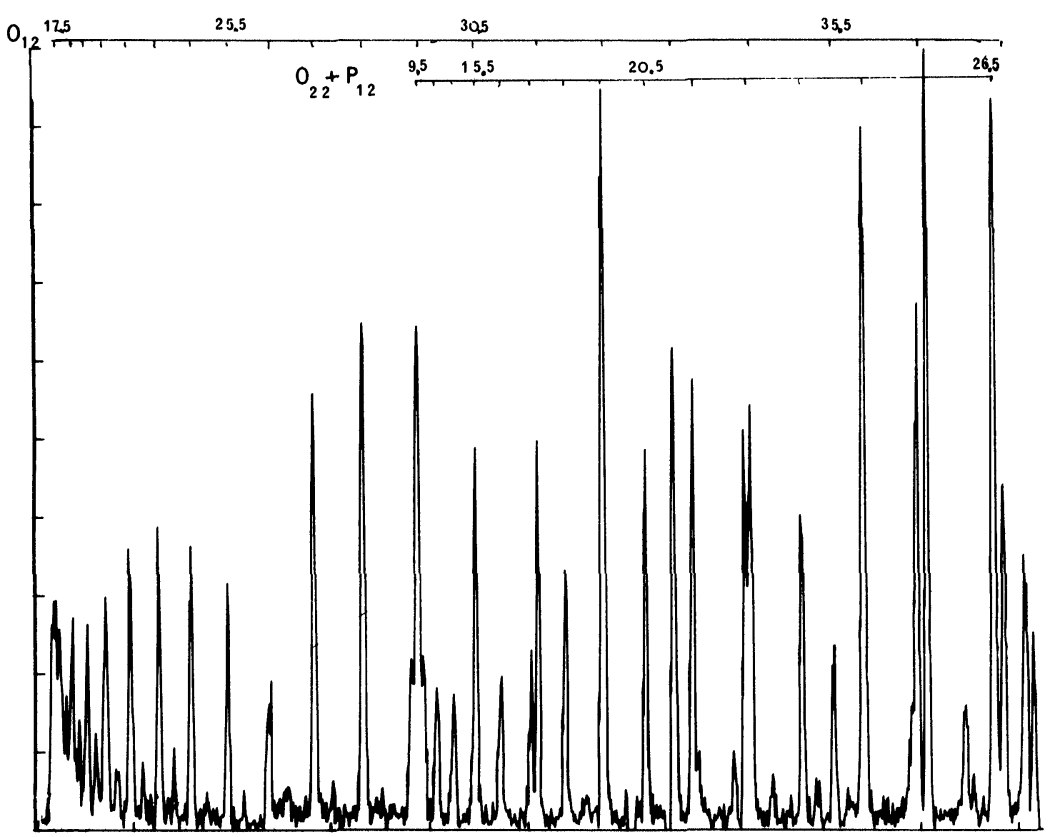

FIGURE 5 Part of the 2 photon excitation spectrum of the NO $X v^{\prime \prime}=1 \rightarrow A v^{\prime}=0$ transition produced in the $3550 \AA$ photodissociation of 100 mTorr of $\mathrm{CH}_{3} \mathrm{ONO}$.

in one hand and NO $X$ at $3550 \AA$ in the other hand. In the former case the rotational energy disposal can be explained by angular momentum conservation while in the latter case the high degree of rotational excitation may come from the torque applied to NO by the recoil of the fragments or from the excitation of torsional modes in the initial molecule. These results emphasize the importance of the electronic excited state surfaces in determining the energy partitioning during the fragmentation.

\section{References}

1. L. Batt, K. Christie, R. T. Milne and H, J, Summer, Int. J. Chem. Kinet. 6, 877 (1974).

2. J. G. Calvert and J. N. Pitts, Photochemistry (John Wiley, New York, 1965) p. 481.

3. K. Ohbayashi, H. Akimoto and I. Tanaka, J. Phys. Chem. 81, 798 (1977). 
4. C. A. F. Johnson, V. Freestone and J. Giovanacci, J. Chem. Soc. Perkin II, 581 (1978).

5. F. Lahmani, C. Lardeux, M. Lavollée and D. Solgadi, J. Chem. Phys. 73, 1187 (1980).

6. H. R. Wendt and H. E. Hunziker, J. Chem. Phys. 71, 5202 (1979).

7. J. B. Halpern, H. Zacharias and R. Wallenstein, J. Mol. Spec. 79, 1 (1980). 